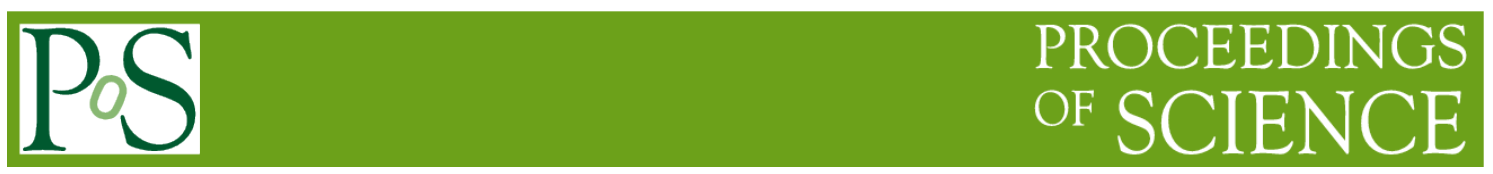

\title{
Concept, Design and Metrology of the WSRT
} Antennas

\section{Jaap Baars}

Max Planck Institut für Radio Astronomie

Auf der Hügel, 53121 Bonn, Germany

E-mail: jacobbaars@arcor.de

\section{Jan Buiter}

ASTRON (retired)

Oude Hoogeveensedijk 4, 7991 PD Dwingeloo, The Netherlands

E-mail: janhilliebuiter@gmail.com

\section{Roelof Kiers}

ASTRON (retired)

Oude Hoogeveensedijk 4, 7991 PD Dwingeloo, The Netherlands

E-mail: roelof.kiers@hetnet.nl 


\section{Chapter 2 Concept, Design and Metrology of the WSRT Antennas}

Jaap Baars*

\section{Enter the BCAP/WSRT Project}

In the spring of 1963 , shortly before my physics graduation at the TU Delft, I applied for a job with the Benelux Cross Antenna Project (BCAP) at Leiden Observatory. The project was somewhat in disarray. Belgium had announced its withdrawal, and a significantly cheaper, and hence smaller, instrument was to be defined. With the successful demonstration of earth-rotation-synthesis by Ryle's group in Cambridge, the "Cross" was being reduced to a "Line" and Jan Högbom, Lex Muller and Ben Hooghoudt were trying to find the best array layout fitting the budget. They did not need me for that, but offered me to join the receiver development group. That was not what I wanted and with Prof. Oort's help I landed a junior research position at NRAO in Green Bank.

Upon my return in the fall of 1966 the telescope had been fully defined and I was given the job of assistant to Ben Hooghoudt, who was Project Manager for the civil works and the mechanical, electrical and control section of the Project. Soon I was spending most of my time at the site in Westerbork, where a lot of different activities were carried out. I was given the task overseeing and coordinating the large amount of metrology activities, and to assure that the contractor would satisfy the specifications. Hooghoudt involved me increasingly in the management of the project.

\section{Specifications of the Westerbork Synthesis Radio Telescope (WSRT)}

The original WSRT consists of 10 polar mounted reflector antennas of $25 \mathrm{~m}$ diameter, placed at an interval of $144 \mathrm{~m}$ along an East-West baseline and a 300 $\mathrm{m}$ long rail track at the eastern end that carries two more mobile antennas. The signals from the two mobile elements are combined ("correlated") with each of the 10 fixed antennas to provide 20 interferometers of different baseline length. During 12 hours observation the projection of the baselines on the sky rotates, providing data in the orthogonal coordinate. Four different positions of the mobile antennas "synthesise" a fully covered telescope of $1600 \mathrm{~m}$ diameter. It yields an angular resolution of 25 arcseconds at $21 \mathrm{~cm}$ wavelength. The observtronomie, Bonn, Germany ing process is slow, but it allows the creation of a large "synthesis telescope" at affordable cost. 
For the proper operation of a synthesis telescope it is important that the positions of the element antennas with respect to each other are accurately known at all times during the long observing time. The same is true for the time differentials that the received signals encounter on their path from the antenna through receiver electronics and cables to the correlator. In an ideal situation, these data are known and stable over the time of observation. This requirement may extend over several days as the mobile antennas occupy different positions on the track.

One way to accommodate deviations from this requirement is to determine the instrumental parameters from an observation of a cosmic radio source with a precise celestial position and sufficient intensity. This is called "calibration" of the interferometer. In the early sixties, not many such sources were available. Also, calibration takes away observing time from the object of interest and requires additional computation in real time. Synthesis is inherently computation intensive and computer power in those days was limited (the IBM 360 only became available in the mid sixties).

The decision was made to realise the synthesis telescope with a geometrical, mechanical and electronic precision and stability that would enable observations without the need for detailed calibration and correction of the data. The major specifications can be summarised as follows:

baseline: East-West to $<1$ arcsecond, straight and flat to $<1 \mathrm{~mm}$

- antenna position: $<1 \mathrm{~mm}$ in all three coordinates

antenna internal dimensions and axis perpendicularity to $<1 \mathrm{~mm}$ and $<5$ arcsec reflector precision $5 \mathrm{~mm} \mathrm{rms}$ for almost perfect performance at $20 \mathrm{~cm}$ wavelength

a high symmetry in all system parts influencing phase stability.

\section{Metrology methods and equipment}

3.1. Baseline and antenna position

The E-W baseline was established by three concrete pillars at both ends and at the mid-point of the baseline. At the nominal position of each antenna, 144 $\mathrm{m}$ apart, a pillar with adjustable marker was aligned to the established $\mathrm{E}-\mathrm{M}-\mathrm{W}$ line. Dr. G. van Herk, a seasoned astrometrist of Leiden Observatory who had spent long periods in his younger years on the equator at a high site in Kenya measuring star positions was assisted by several colleagues to perform the measurements. Van Herk preferred drizzly weather because of the more stable atmosphere. He withstood the rain by cutting some holes for his arms and head in a huge plastic garbage bag, which we then pulled over his head at the start of the shift. He established the azimuth and declination of the baseline to exactly 90 and $\circ$ degrees, respectively with an uncertainty of 0.5 arcsec. Later astronomical calibration confirmed the azimuth within the error and indicated a declination of -0.6 arcsec. The position of the markers at the antenna posi- tion was determined with an accuracy of $0.1-0.3 \mathrm{~mm}$. Standard geodetic methods were used to position the antenna with respect to these markers. Radio astronomical checks found a few antennas to deviate by $2-3 \mathrm{~mm}$, the remaining were within the measurement error of $0.5 \mathrm{~mm}$

3.2. Assembly of the antenna structures

A large temperature controlled assembly hall was erected at the site in which templates were placed to aid in the welding and assembly of the major sections of the antenna. These are sketched in Figure 1 and the hall is shown in Figure 2. A challenge was the realisation of better than $1 \mathrm{~mm}$ precision in the important dimensions within the antenna structure and amongst the 12 antennas, while allowing "standard" workshop procedures as far as possible. The shop fabrication produced parts that could be transported to the site. The biggest pieces were the gear racks that came by ship to about $10 \mathrm{~km}$ from the site and needed special road transfer for the last leg. The polar axis house and declination cradle (see Figure 1) were welded in templates with a carefully controlled procedure, continuously checked by measurements. The same expert welders worked on this during the entire production. They assured identical dimensions among the 12 antennas. The orthogonality of polar and declination axes is better than $0.2 \mathrm{~mm}$ and 3 arcsec.

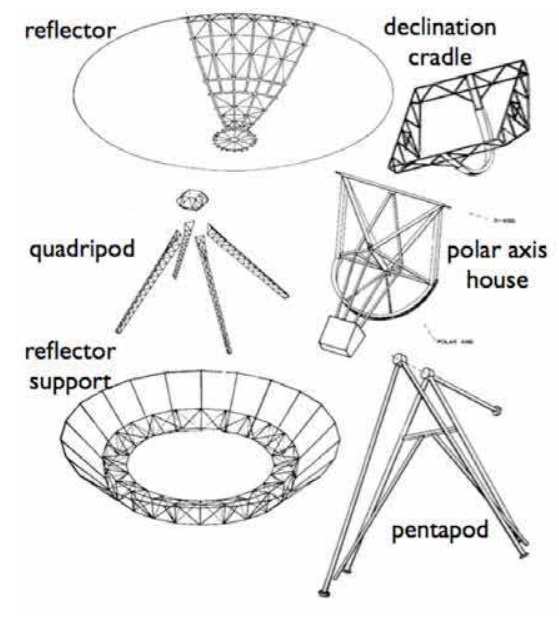

Figure 1. Exploded view of the major antenna sections. Polar axis house and declination cradle are welded in accurate templates.

The assembly of the reflector was the most time consuming and labour intensive activity. Mr. A. Bijloo of Wilton-Feijenoord developed the template layou and the assembly and measurement procedure. As shown in Figure 2, the template consists of 72 vertical steel frames that carry the support pins on which the reflector surface panels will be laid out (Figure 3). There are 48 panels in 


\section{PLAN VIEW}
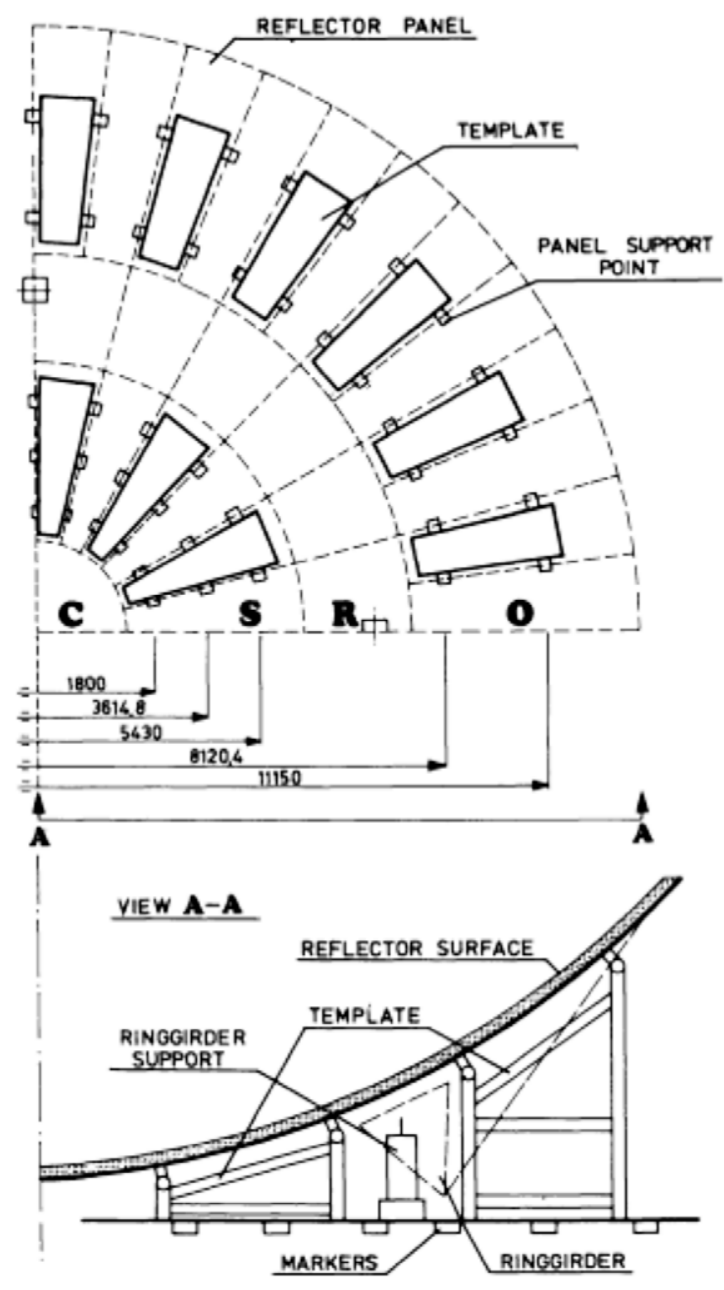

the outer and 24 in the inner ring. Within the template there are 4 support columns that mimic the four corners of the declination cradle, to which the ring girder of the reflector is attached. The ring girder is welded complete from the four sections delivered from the factory.

The support pins on the template frames are adjusted to the required position by optical means as illustrated in Figure 4 . A levelling instrument determines the correct height with respect to a calibration pillar and the radial coordinate is set with the aid of an optical plumb line to markers on the floor. Note that no angle measurements are involved in this scheme. The position of the support pins is precise to a few tenths of a millimetre. For this tedious and difficult work Bijloo had the assistance of the twin-brothers Bakker, who later became a
Figure 4

Schematic of

determining the the panel suppor precise position of

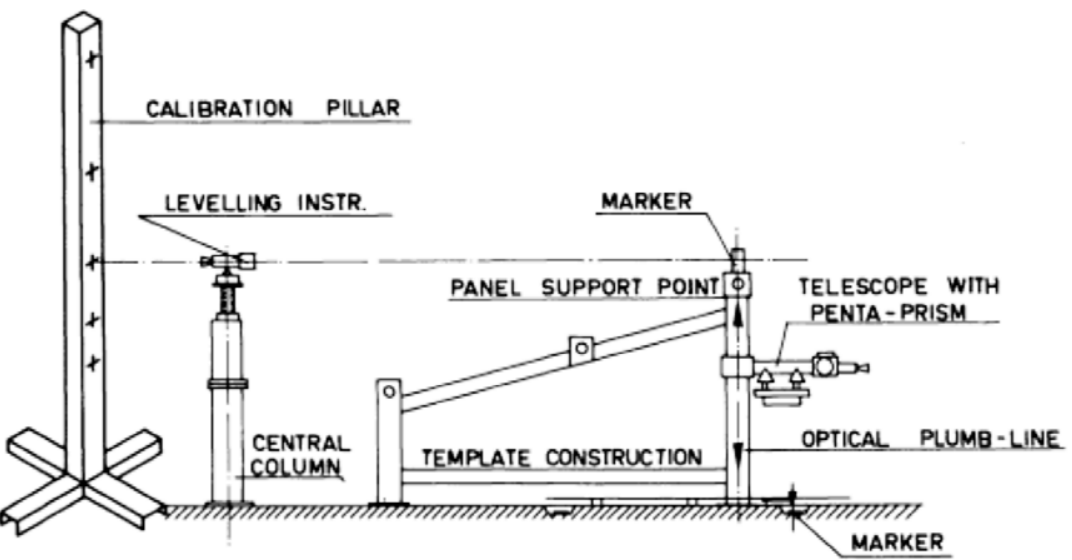

Figure 5. The twin Jaap Bakker adjusting a support pin. The author observes and documents the readings.

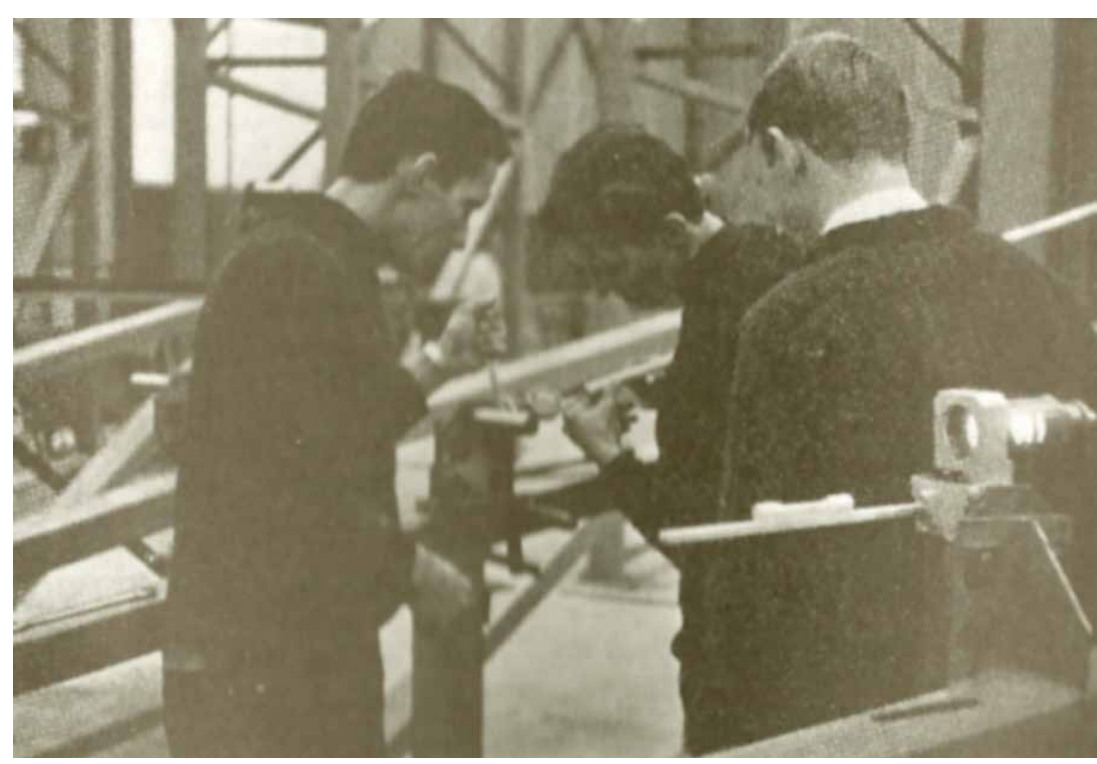

staple of the mechanical workshop in Dwingeloo. They formed a remarkable team. Once Bijloo described to them the need for a tool to simplify the work. They listened, nodded, and without exchanging a word each went to a different machine or bench. After an hour they produced the tool consisting of severa parts that fitted together perfectly. Figure 5 shows them at work while I record their measurements on paper.

3.3. Assemble reflectors

The reflector panels, each a stiff steel frame of parabolic shape with a stainless steel surface mesh bonded by epoxy resin, were laid out on the support pins. The same measurement method was used to check the height of the surface in 


\section{The I968 Building Site}

Ian Buiter, Roelof Kiers

Quoting from Anke den Duyn's*: text in the ASTRON JIVE Daily Im age, the shortest story of the beginning of WSRT is as follows: At the silent places on Earth, where astronomers look back in time with advanced technology various historical timelines may intersect. In 1964 an con of radio astronomy was put on the map by means of a thin red line crossing out camp "Schattenberg". It was the first uncontroversial use of this small piece of land for several decades. More than ten years earlie, in March 1951, the ship "Kota Inten" had brought the first KNIL soldier and their families from the tropical Molucca Islands to the chilly Nether-
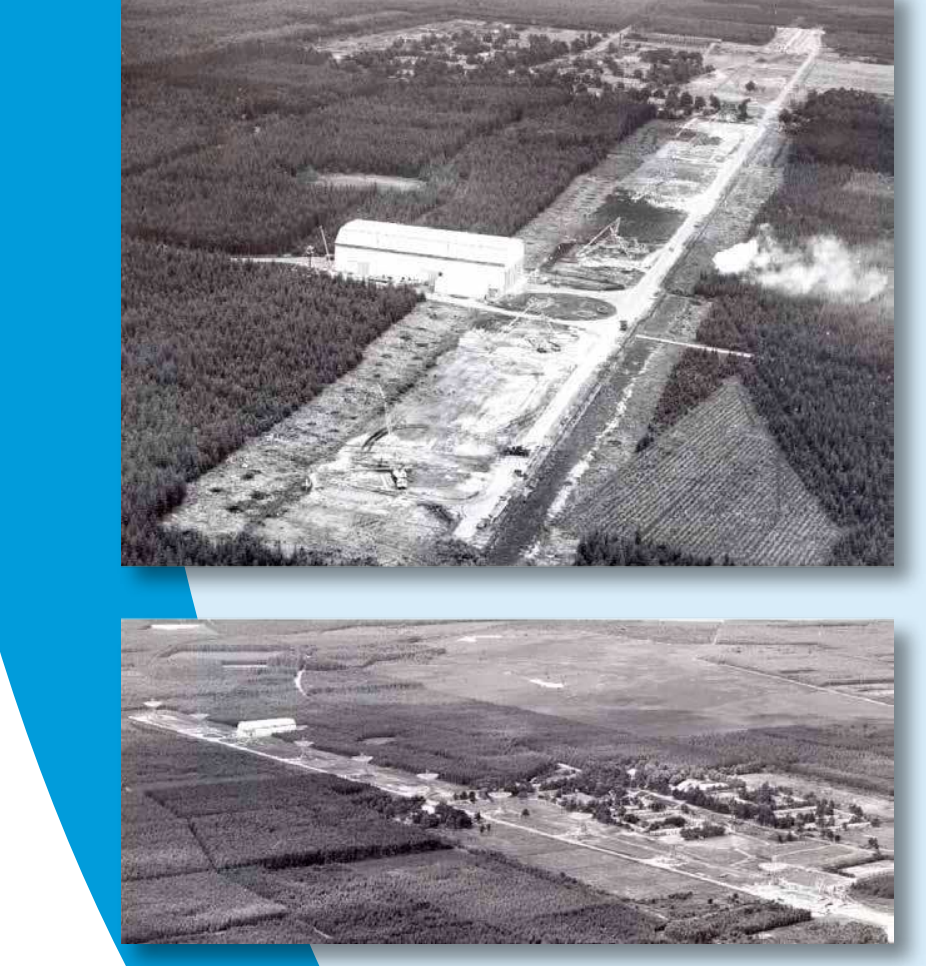

lands just before Indonesia as former Dutch colony won its independence. They were accommodated into the former transit camp Westerbork now renamed Schattenberg.

In 1966 about two years after breaking soil for the building of the WSRT the situation was as shown in the picture. With the assembly hall in the foreground between the yat unfished telescopes RT and RT2 and the foundations of RTo in the near foreground, it also shows in the background the Schattenberg camp which was in the slow process of being dismantled. In fact, as the WSRT building evolved and started to do its earliest operations in 1968, a few hundred men, women and children were still living on the site watching the machine of the future being built. Co-existence evolved smoothly while the relocation progressed. The first engineers, observers, and operators on site fondly remember the Moluccan specialties kindly offered to them by their neighbours during 24/7 observing duties! They included Jan Buiter, one of the early Dwingeloo engineers involved in the construction, and observers/operators Sip Siitsma and Kees Brouwer, and later Roelof Kiers who joined in 1970. Sip Sljtsma remained in Westerbork while the other three joined the lab in Dwingeloo and continued working for ASTRON until their retirement.

Anke den Duyn is author of the book "Het logboek" which appeared in 2014 and translated as "The Journa" in 2016 (ISBN 9789082239614$)$ order to correct imperfections in the panel with spacers. Once the panels were properly positioned they were bolted together to form the final surface. The panels provide the required stiffness; no further support structure is needed for the inner panel ring. The outside ring is supported by radial beams connected by a hoop at a radius of $10 \mathrm{~m}$ (Figures 1 and 6 ).

The connection of the reflector with the ring girder is highly original and simple. At each connection point there is a socket on the ring girder and the pane has a downward pin that protrudes into the socket with plenty of lateral tolerance to accommodate for standard manufacturing tolerances during welding of the ring girder in the shop. The space between pin and socket is filled with epoxy resin. A stress free connection is formed thereby without any influence on the reflector. This innovative solution has endured 50 years without any noticeable degradation! Unfortunately the epoxy bonding of the panels broke after about 8 years and was solved by fixing the mesh to the frame with thousands of screws (see insert by Wout Beerekamp). The method was later applied at other telescopes and with the improved precautions, proved successful.

The completed reflector surface contour was measured at about 250 points on the surface to check the overall parabolic shape. These measurements indicated some small systematic deviations, mainly in the outer panel ring, that could be decreased by changes in the order of bolting the panels. The average surface error of the antennas is $1.5 \mathrm{~mm} \mathrm{rms}$ which is a factor three better than the specification. The very low surface error was achieved thanks to the high precision of the individual panels, and also to the corrections applied during the carefully measured assembly procedure.

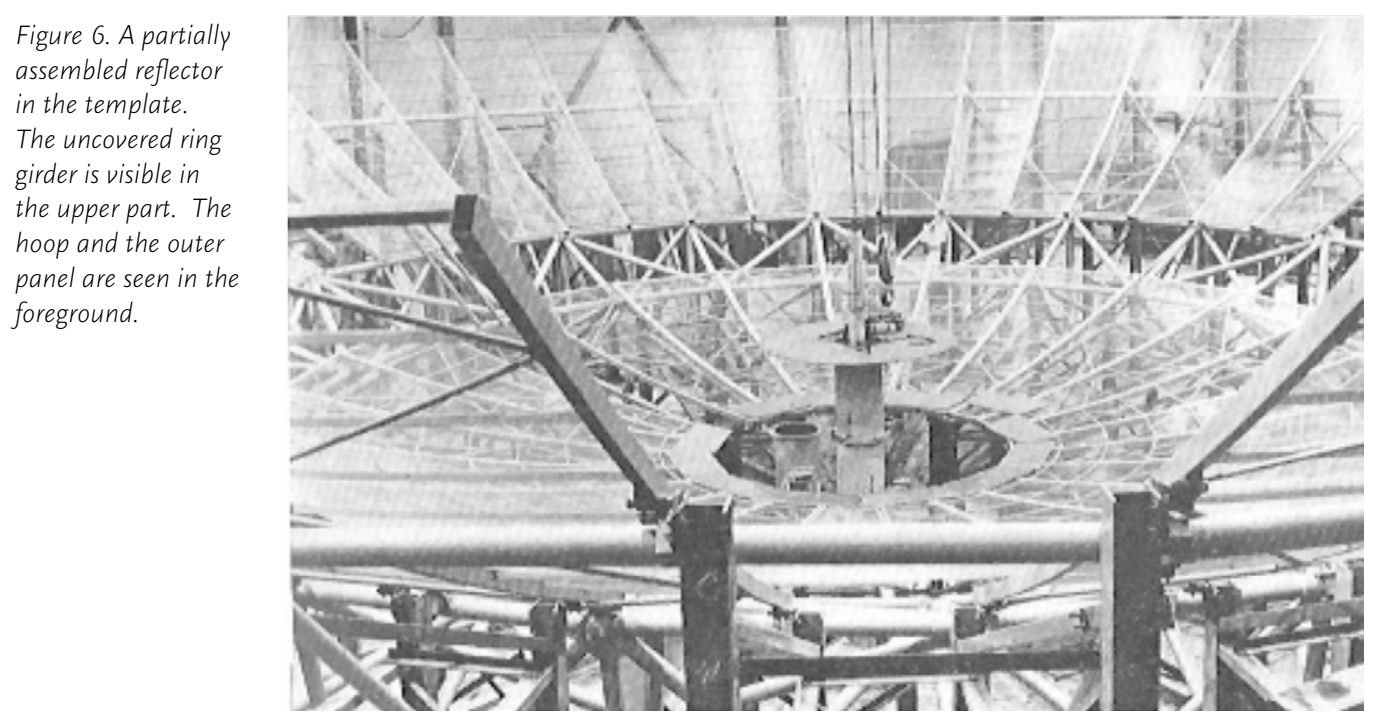


A further test was done to measure gravitational deformation. By lifting the reflector off the template so that it was supported only on the four support pillars of the ring girder, the gravitational deformation in zenith position was determined. It turned out to be about half the predicted value from the structural analysis, making the careful assembly all the more valuable. The result was that the telescope could be used with high efficiency at $6 \mathrm{~cm}$ wavelength.

\section{Conclusion}

Looking back 50 years I find it remarkable that all involved maintained a dedication to achieving the best possible precision in a repetitive process of assembling 12 antennas over a period of close to two years. Not much exciting happened on site, so we all took a brake when the huge crane appeared to assemble two antennas within three days. We had a really scary moment during the hoisting of the first reflector. A manoeuvre had been worked out to use two relatively small cranes, one of which was already in continuous use on site. Early in the hoisting with both cranes moving the reflector between them the load balance got skewed and a cable broke. With great luck we managed to lower the dish to the ground without damage. The rest of the hoists were performed with one huge crane with a double boom. It was expensive but it worked flawlessly.

One can ask whether the very tight tolerances applied to about every aspect of the WSRT, not only the baseline and antennas but also the electronic and cable system, weren't a bit of overkill. In the current situation of abundant computing power one might not be so demanding about mechanical tolerances. I have asked a few colleagues of the early days this question and their answer is “no, it wasn't overkill!” The high precision and, perhaps more important, the very high stability of the baseline while tracking enabled 12-hour observations without interruption for calibration. It helped the commissioning enormously and good quality maps were obtained from the beginning.

I conclude that we did not waste our time during the construction period. I add that it was also a period of satisfying work with excellent collaboration between observatory staff and contractors. The leadership of Ben Hooghoudt was stern but fair and he ensured that the relationship between customer and contractor did not become too cosy. 